\title{
PROJETO E OTIMIZAÇÃO DE REATOR DE DESSULFURAÇÃO DO GUSA ATRAVÉS DE MODELO DE TRANSPOSIÇÃO DE RESULTADOS
}

\author{
Sérgio Luiz de Souza Costa ${ }^{1}$ \\ Ivan Lopes Alves ${ }^{2}$ \\ Jorge Lima de Siqueira ${ }^{3}$
}

\section{Resumo}

A dessulfuração de gusa foi otimizada pela adoção do processo Cal-Mg. Nesse processo, o magnésio é utilizado para reduzir o teor de oxigênio dissolvido para próximo de zero e, assim, o poder dessulfurante da cal é aumentado, o que explica a eficiência do processo. Nesse estudo, avaliou-se a utilização da lança rotativa em substituição à atual lança de injeção de mistura de material dessulfurante na Aciaria I da Usiminas. Além disso, desenvolveu-se um critério, que leva em conta as dimensões do equipamento e as taxas de injeção de gás e de material dessulfurante, para transposição dos resultados para plantas de dessulfuração de gusa de maior dimensão. A lança rotativa promoveu uma melhor distribuição do material dessulfurante, obtendo-se uma elevação na taxa de dessulfuração de $18 \%$ e diminuição de projeção de metal. A transposição dos resultados de dessulfuração de gusa para uma planta de maior porte foi realizada com sucesso, o que permite concluir que o critério desenvolvido pode ser usado para a tomada de decisão quanto à implantação da lança rotativa em outras plantas industriais, sem a necessidade de realização de experiências. $O$ critério desenvolvido também pode ser utilizado em estudos de otimização de outras plantas industriais.

Palavras-chave: Processo Cal-Mg; Dessulfuração; Lança rotativa.

\section{PROJECT AND OPTIMIZATION OF HOT METAL DESULPHURIZATION REACTOR BY USING TRANSPOSITION RESULTS MODEL}

\begin{abstract}
The hot metal desulfurization has been speeded up by adoption of lime-Mg process. In this process, the magnesium is used to reduce the oxygen content in the hot metal to almost zero, increasing the deS power of the lime, thus explaining the efficiency of such process. In the present study, preliminary experiments were carried out in cold models, using rotating lance, which resulted in a better material distribution. The rotating lance tested in the hot metal desulphurization plant of Usiminas' Steel Plant I promoted a 18\% increase in the desulphurization rate, a 54\% decrease in temperature drop during the treatment, as well as a reduction of metal splash occurrences. It was applied a transposition model, whose criteria takes into account the equipment dimensions and the mass flow rate of the desulphurization material. In order to evaluate the applicability of the this model, the results of hot metal desulphurization obtained in a $100 \mathrm{~kg}$ atmospheric induction furnace were transposed to a $160 \mathrm{t}$ torpedo car. The theoretical desulphurization results were very close to the measured values. It was possible, from these results, to predict the desulfurization rate, the material consumption and the treatment time, which can indicates the technical and economical viability of the rotating lance.
\end{abstract}

Key words: Lime-Mg process; Desulfurization; Rotating lance.

\section{INTRODUÇÃO}

$\mathrm{Na}$ fabricação do aço, o pré-tratamento do gusa é de fundamental importância para a obtenção de produtos de melhor qualidade. A busca de eficiência nesse processo tem resultado em novos procedimentos e inovações nos equipamentos.
No tratamento de dessulfuração do gusa promove-se a injeção de pós dessulfurantes, no interior do metal líquido, utilizando-se lanças refratárias e um gás de arraste, normalmente o nitrogênio. Tais lanças são dotadas de movimento apenas vertical e têm por finalidade a injeção de

\footnotetext{
' Engenheiro Metalurgista, Dr., Centro de Pesquisa e Desenvolvimento da Usiminas; Ipatinga, MG.

2 Técnico Metalurgista, Gerência de Convertedor da Aciaria I da Usiminas; Ipatinga, MG.

${ }^{3}$ Técnico Metalurgista; Assistente Técnico da Insider, Cel Fabriciano- MG.
} 
pós a uma maior profundidade possível, com pressão e vazão limitadas para se evitar desgaste prematuro do fundo da panela e o transbordamento de metal líquido. Visando, principalmente, minimizar esses problemas, foi realizada uma avaliação qualitativa da injeção de pós, em um modelo físico, utilizando-se uma lança com dois furos horizontais, dotada de movimento de rotação em torno do seu eixo, e comparada com a utilização de uma lança fixa. A melhor distribuição de linhas de fluxo, obtida com a lança rotativa, motivou a realização de experiências industriais na Aciaria I da Usiminas.

No presente estudo, através de um trabalho conjunto Usiminas/Insider, foi avaliada a eficiência da utilização da lança rotativa no pré-tratamento de dessulfuração do gusa, em panela pelicano, por meio do processo Cal-Mg. Além disso, aplicou-se um modelo de transposição desenvolvido por $\operatorname{Costa}^{(1)}$ que permite prever os resultados de dessulfuração de gusa em outras plantas industriais de diferentes dimensões.

\section{EXPERIÊNCIAS REALIZADAS NA PLANTA DE DESSULFURAÇÃO DE GUSA DA ACIARIA I}

\section{I Modelo Físico}

Os primeiros testes consistiram de uma avaliação qualitativa da injeção de pós, em um modelo físico, construído em escala I:5, utilizando-se uma lança com dois furos horizontais, dotada de movimento de rotação em torno do seu eixo, e comparada com uma lança fixa. A melhor distribuição de linhas de fluxo, obtida com a lança rotativa, motivou a realização de experiências industriais na Ac. I da Usiminas.

Esses resultados levaram ao desenvolvimento de uma lança rotativa, em escala industrial, com o objetivo de se realizar testes industriais, cujos resultados evidenciaram a promissora eficiência da lança rotativa na dessulfuração do gusa.

\subsection{Descrição Geral do Funcionamento da Lança Rotativa}

O sistema consiste de uma junta rotativa que permite $o$ acoplamento da lança com uma mangueira de injeção de gás e pó sem que esta gire junto com a lança. A lança é revestida com refratário e possui dois orifícios na extremidade para injeção de dessulfurante. $O$ moto redutor é equipado para promover a regulagem da rotação da lança durante o seu movimento de descida. O carro de sustentação permite a imersão da lança no metal. Este carro possui inversores de freqüência que promovem o controle da velocidade de subida e de descida da lança.

O equipamento possui um sistema de troca rápida da lança refratária em caso de entupimento. Na parte inferior do conjunto existe uma caixa revestida de isolamento térmico que impede a transferência de calor para o equipamento, a fim de proteger a lubrificação das engrenagens e rolamentos. A lubrificação do equipamento é executada através de pinos graxeiros, localizados na parte externa do sistema, sem ter que se desmontar o equipamento.
O equipamento é montado e alinhado de forma que o centro da lança coincida com o centro da panela. A lança deve ficar a uma distância de $300 \mathrm{~mm}$ da sola da panela quando estiver no seu ponto máximo inferior e a $1.000 \mathrm{~mm}$ fora da panela no seu ponto máximo superior. Tal controle é feito através dos limitadores de curso que interrompem a energia nos motores dos carros de elevação.

\subsection{Condições Experimentais}

Numa primeira etapa foram acompanhados 24 tratamentos de dessulfuração de gusa, sendo 12 com utilização da lança rotativa e 12 com a lança convencional. Nessas experiências avaliaram-se os teores iniciais e finais de enxofre, a queda de temperatura, o desgaste de refratário da lança e o nível de projeção de metal para se ter uma comparação da eficiência global dos dois processos.

Numa segunda etapa foram acompanhados 25 tratamentos de dessulfuração de gusa na Ac. I, retirando-se amostras em intervalos de um minuto, ao longo de todo o tratamento com lança rotativa. Essas amostras foram analisadas e, com base nos teores de enxofre, determinou-se o coeficiente de transferência de massa. A partir desse coeficiente, efetuou-se uma transposição dos resultados para uma planta de dessulfuração de gusa com dimensões diferentes. Os principais parâmetros de projeto e operacionais da planta de dessulfuração de gusa da Aciaria I e das dimensões consideradas na transposição são apresentados na Tabela I.

Tabela I. Grandezas relacionadas com o equipamento da Aciaria I e com as simulações.

\begin{tabular}{|c|c|c|}
\hline Parâmetros & Aciaria I & Simulações \\
\hline Diâmetro da panela (m) & 2,8 & 3,5 \\
\hline Altura da panela $(\mathrm{m})$ & 3 & 3,8 \\
\hline Peso de gusa $(\mathrm{t})$ & 65 & 160 \\
\hline $\begin{array}{l}\text { Vazão de gás de arraste } \\
\left(\mathrm{Nm}^{3} / \mathrm{h}\right)\end{array}$ & 15 & 60 a 120 \\
\hline $\begin{array}{l}\text { Taxa de injeção de } \\
\text { magnésio }(\mathrm{kg} / \mathrm{min})\end{array}$ & 6 & 10 a 20 \\
\hline $\begin{array}{l}\text { Taxa de injeção de cal } \\
(\mathrm{kg} / \mathrm{min})\end{array}$ & 24 & 40 a 80 \\
\hline Borda livre (m) & 0,8 & 0,44 \\
\hline Temperatura $\left({ }^{\circ} \mathrm{C}\right)$ & 1.300 a 1.350 & 1.300 a 1.350 \\
\hline Enxofre inicial (\%) & 0,025 a 0,045 & 0,025 a 0,045 \\
\hline
\end{tabular}

\section{CRITÉRIO GERAL PARA TRANSPO- SIÇÃO DOS RESULTADOS}

De um modo geral, pode-se dizer que toda a divergência, reportada na literatura, entre resultados de laboratório e escala industrial, em 
tratamentos de metal líquido, se deve a fatores cinéticos, diretamente ligados ao nível de agitação do banho. É consenso na literatura que a eficiência de todos os processos metalúrgicos industriais, envolvendo reações químicas, é fortemente dependente taxa de dissipação de energia, ${ }^{(1)}$ representado por $\varepsilon(\mathrm{W} / \mathrm{t})$.

$O$ efeito das dimensões do reator tem sido considerado, embora não exista consenso sobre como expressar sua influência. ${ }^{(2-6)}$ Pode-se ver, ainda, que os critérios disponíveis na literatura citada são válidos só para faixas específicas de tamanho de reator, e que uma transposição confiável e geral de dados, da escala de laboratório para a industrial, com base nesses critérios, não é possível.

A Tabela 2 apresenta um resumo das diversas relações de transposição de resultados propostas na literatura, com as dimensões e os campos de validade.

Tabela 2. Resumo das relações de transposição disponíveis na literatura.

\begin{tabular}{|c|c|c|}
\hline Pesquisador & Relação & Validade \\
\hline Nakanishi $^{(3)}$ & $\tau \alpha \varepsilon^{-0,4}\left(m^{-0,8} s^{1,2}\right)$ & $\begin{array}{l}\text { Pequenas mudanças de es- } \\
\text { cala ( } L \text {, } D \text { e } \varepsilon \text { ). Significado } \\
\text { físico não é claro. }\end{array}$ \\
\hline $\begin{array}{l}\text { Ohguchi e } \\
\text { Sambongi( }{ }^{(4)}\end{array}$ & $\mathrm{k} \alpha \varepsilon^{1 / 2}\left(\mathrm{~m} \cdot \mathrm{s}^{-3,2}\right)$ & $\begin{array}{l}\text { Pequenas mudanças de es- } \\
\text { cala ( } L \text {, } D \text { e } \varepsilon \text { ). Significado } \\
\text { físico não é claro. }\end{array}$ \\
\hline Watanabe $^{(5)}$ & $k \alpha \varepsilon^{1 / 3}\left(m^{2 / 3} s^{-1}\right)$ & $\begin{array}{l}\text { Ajustou a influência de } \varepsilon \text {. } \\
\text { Ignora efeito das dimen- } \\
\text { sões do equipamento. Váli- } \\
\text { da para pequenas variações } \\
\text { de tamanho do reator. }\end{array}$ \\
\hline Sano e Mori $i^{(6)}$ & $\tau=100\left[\left(\mathrm{D}^{2} / \mathrm{L}\right)^{-2}\right]^{-0,337}\left(\mathrm{~m}^{0,67}\right)$ & $\begin{array}{l}\text { Para previsão de tempos de } \\
\text { mistura perfeita em peque- } \\
\text { nos reatores e modelos a } \\
\text { frio. Significado físico não } \\
\text { é claro. }\end{array}$ \\
\hline Asai e Muchi ${ }^{(2)}$ & $k \alpha\left(\varepsilon L^{3} / D^{2}\right)^{1 / 3}(m / s)$ & $\begin{array}{l}\text { Processos pouco agita- } \\
\text { dos; na macroescala de } \\
\text { turbulência. Expressa a } \\
\text { velocidade de escoamento } \\
\text { na micro-escala de tur- } \\
\text { bulência. }\end{array}$ \\
\hline Kitamura et al. ${ }^{(7)}$ & $k \alpha \varepsilon L^{2} / D\left(m^{3} s^{-3}\right)$ & $\begin{array}{l}\text { Grandes mudanças de es- } \\
\text { cala. Significado físico não } \\
\text { é claro. }\end{array}$ \\
\hline
\end{tabular}

* $\mathrm{L}$ - altura da panela; $\mathrm{D}$ - diâmetro da panela; $\varepsilon$ - taxa de dissipação de energia; $\mathrm{k}$ - coeficiente de transferência de massa; e $\tau$ - tempo requerido para misturamento perfeito.

Nos processos com escoamento turbulento a etapa controladora é a transferência de momento e massa, que irá ocorrer na micro-escala de turbulência, onde predominam as forças viscosas. É importante observar que a presença do expoente $1 / 3$ está em concordância com diversas correlações experimentais. $(2,5,6)$ Tal expoente é justificado pelas condições de fluxo nos diversos processos industriais e de laboratório, cujas taxas de dissipação de energia são superiores a $4 \mathrm{~W} / \mathrm{t} .{ }^{(8)}$ Tal fato indica que o controle da mistura se dá pela difusão de redemoinhos. ${ }^{(8)}$ Como o critério proposto por Asai e Muchi ${ }^{(2)}$ tem dimensão de $\mathrm{m} / \mathrm{s}$, ele representa a velocidade de escoamento na micro-escala de turbulência, ou seja, a convecção do metal líquido em estado estacionário. Nesse sentido, seria válido como critério de transposição só para processos com taxa de dissipação de energia inferiores a $4 \mathrm{~W} / \mathrm{t}$, nos quais as transferências de massa e momento são controladas pelas forças de inércia.

Tomando como base o trabalho de Asai e Muchi ${ }^{(2)}$, Costa ${ }^{(1)}$ propôs um critério geral de transposição de resultados, tendo em vista as limitações encontradas na literatura. De acordo com Szekely e Themelis, ${ }^{(9)}$ o fenômeno de transferência de massa ocorre em duas etapas: nas macroescalas de turbulência predominantemente por convecção e ao atingir as micro-escalas de turbulência por difusão. Para as micro-escalas, onde as forças viscosas são predominantes, têm-se:

$$
\mathrm{N}_{\mathrm{Re}}=\frac{\mathrm{D} v^{*}}{v^{*}}=1
$$

onde $v$ é a velocidade de escoamento no interior do redemoinho e $v$ * o coeficiente de transferência de momento por difusão na micro-escala, sendo $v$ deduzida anteriormente por Asai e Muchi, ${ }^{(2)}$ conforme apresentado na Tabela 2:

$$
v^{*} \alpha\left[\frac{\varepsilon^{\frac{1}{3}} L}{D^{\frac{2}{3}}}\right]
$$

Substituindo a Equação 2 em I, Costa ${ }^{(1)}$ obteve a seguinte equação:

$$
v^{*} \alpha\left(\mathrm{DL}^{3} \varepsilon\right)^{\frac{1}{3}}
$$

Baseando-se na analogia entre transporte de momento e massa, pode-se afirmar que tal equação representa um critério geral de transposição em processos controlados por difusão de massa e momento, na micro-escala de turbulência. Com dimensão de $\mathrm{m}^{2} / \mathrm{s}$, o critério representa a própria difusividade no redemoinho.

Para que a reação química possa ocorrer, os reagentes devem, inicialmente, ser transportados para a frente de reação. Como tal transporte ocorre em duas etapas, qualquer critério deve levar em conta o tempo necessário para: primeiro, partindo da macroescala de turbulência (depende das dimensões do reator) até atingir a microescala e, segundo, o tempo necessário para a difusão desses microredemoinhos (função da taxa de dissipação de energia). Portanto, as dimensões do equipamento precisam ser também consideradas para que as diferenças de tempo gasto para se atingir a micro-escala de turbulência, nos dois casos, não sejam desprezadas.

Os valores do coeficiente de transferência de massa (k) em escala de laboratório podem ser determinados experimentalmente. Os valores de 
taxa da dissipação de energia nas duas escalas são também conhecidos. Assim, a transposição para determinação de $\mathrm{k}$ na instalação industrial é diretamente proporcional à escala, que, no caso de uma planta de dessulfuração, deve ser corrigido levando em conta - peso de dessulfurante injetado por tonelada de gusa nos dois equipamentos. Assim, a evolução teor de enxofre ao longo do tratamento pode ser facilmente determinada.

Tal critério pode ser entendido como o resultado da seqüência de etapas, que envolvem o mecanismo de transferência dos reagentes, conforme descrito a seguir nas etapas I e II, anteriores à reação química, descrita na etapa III.

- Etapa I - na macroescala de turbulência, onde as forças de inércia prevalecem $(\operatorname{Re}>1)$, o processo é predominantemente convectivo; daí a dependência das dimensões do equipamento;

- Etapa II - na micro-escala de turbulência, onde prevalecem as forças viscosas $(\operatorname{Re} \leq 1)$, o processo é predominantemente difusivo. Assim, o tempo para difusão dos reagentes irá depender do tamanho da micro-escala (diâmetro do redemoinho), daí a dependência da taxa de dissipação de energia; e

- Etapa III - sendo a cal o agente dessulfurante, ${ }^{(10)}$ seu suprimento na frente de reação precisa ser considerado. Isso foi feito levando em conta o consumo de dessulfurante por tonelada de gusa de cada reator.

\section{RESULTADOS E DISCUSSÃO}

\section{I Experiências Industriais na Aciaria I}

Os melhores resultados foram obtidos utilizando-se lança de dois furos com $9 \mathrm{~mm}$ de diâmetro, rotação de $10 \mathrm{rpm}$ e pressão nos vasos de 7 bar.

Tabela 3. Resultados de testes experimentais com Lança Rotativa na Aciaria I.

\begin{tabular}{lccc}
\hline \multirow{2}{*}{ Item } & \multicolumn{3}{c}{ Processo } \\
\cline { 2 - 4 } & Convencional & Lança rotativa & $\Delta(\%)$ \\
\hline Queda de Temperatura $\left({ }^{\circ} \mathrm{C}\right)$ & 20 & 10,9 & 55 \\
Taxa de De-S $(\%)$ & 72 & 85 & 18 \\
Tempo de Tratamento $(\mathrm{min})$ & 8 & 6,4 & 20 \\
Consumo de Cal $(\mathrm{kg})$ & 396 & 317 & 20 \\
Consumo de $\mathrm{Mg}(\mathrm{kg})$ & 88 & 77 & 12,5 \\
\hline
\end{tabular}

Os resultados preliminares permitiram concluir que a lança rotativa é mais eficiente do que a convencional. Nos tratamentos realizados, obteve-se taxa de dessulfuração $18 \%$ maior, aumento de $71,9 \%$ para $84,9 \%$ e redução na queda de temperatura de $54,5 \%$, de $20^{\circ} \mathrm{C}$ para $10,9^{\circ} \mathrm{C}$. Além disso, verificou-se uma menor projeção de metal líquido. Tais resultados foram explicados em função de uma melhor distribuição das linhas de fluxo, como também verificado no modelo físico, o que proporciona uma redução de zonas mortas e faz com que o agente dessulfurante entre em contato com porções renovadas de gusa, tornando este processo mais eficiente.

\subsection{Determinação do Coeficiente de Transferência de Massa}

A Figura I apresenta a evolução do teor de enxofre em função do tempo de tratamento utilizando lança rotativa. Determinou-se o coeficiente de transferência de massa, para cada tratamento, com base nesses dados. De um modo geral, os coeficientes de transferência de massa calculados apresentam pequenas variações e, portanto, representam bem o processo. Essa pequena variação permite realizar simulação para teores de enxofre inicial superiores aos avaliados nesta experiência.

De posse do coeficiente de transferência de massa, o teor de enxofre pode ser estimado, a partir da equação de difusão de primeira ordem, sendo $\mathrm{k}$ o coeficiente global de transferência de massa.

$$
\frac{\mathrm{dS}}{\mathrm{dt}}=\mathrm{k}[\mathrm{S}] \quad \therefore \quad \mathrm{S}=\mathrm{S}_{0} \mathrm{e}^{-\mathrm{kt}}
$$

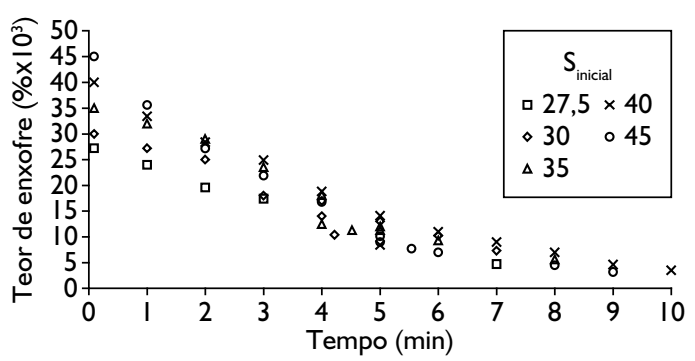

Figura I. Evolução do teor de enxofre medido ao longo do tratamento.

\subsection{Aplicação do Modelo de Transposição}

A Figura 2 apresenta a validação do modelo de transposição proposto por Costa ${ }^{(1)}$ para dessulfuração do gusa em carro-torpedo a partir de testes experimentais num forno atmosférico de indução de $100 \mathrm{~kg}$. Pode-se ver uma concor-

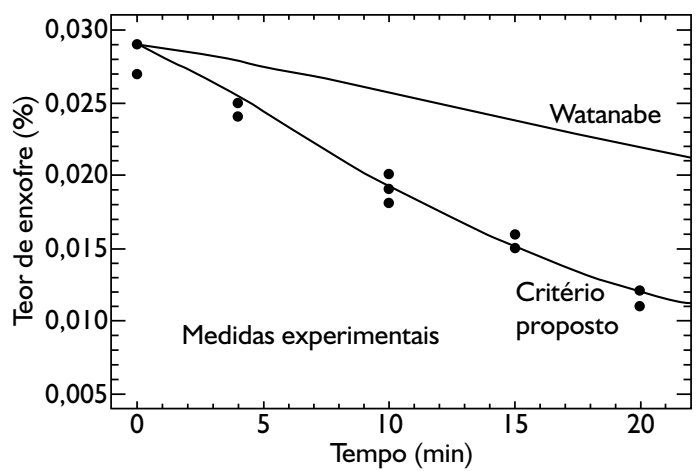

Figura 2. Evolução dos teores de enxofre no carro-torpedo (Critério de Watanabe). ${ }^{(5)}$ 
dância muito boa entre os valores teóricos e os experimentais. Pode-se ver ainda que em equipamentos como o carro-torpedo, a desconsideração do efeito das dimensões do equipamento, conforme Watanabe ${ }^{(5)}$ leva a um resultado muito diferente dos valores obtidos no tratamento industrial.

Os bons resultados obtidos na transposição para o carrotorpedo são a garantia da aplicabilidade do critério de transposição proposto. Efetuou-se sua transposição para uma planta de dessulfuração de gusa hipotética, conforme Tabela 4. Além desses dados, consideraram-se os dados de simulação, conforme Tabela I.

Tabela 4. Planejamento de simulações de transposição.

\begin{tabular}{|c|c|c|c|c|c|c|}
\hline \multirow[t]{2}{*}{ Simulações } & \multicolumn{3}{|c|}{$\begin{array}{c}\text { Simulação I } \\
\left(\mathrm{S}_{0}=\mathbf{0 , 0 7 6 \% )}\right.\end{array}$} & \multicolumn{3}{|c|}{$\begin{array}{c}\text { Simulação II } \\
\left(S_{0}=0,037 \%\right)\end{array}$} \\
\hline & I & 2 & 3 & $\mathbf{I}$ & 2 & 3 \\
\hline Vazão $\left(\mathrm{Nm}^{3} / \mathrm{h}\right)$ & 60 & 90 & 120 & 60 & 90 & 120 \\
\hline Taxa de injeção $M g(k g / m i n)$ & 10 & 15 & 20 & 10 & 15 & 20 \\
\hline Taxa de injeção cal (kg/min) & 40 & 60 & 80 & 40 & 60 & 80 \\
\hline Consumo total de dessulfurante $(\mathrm{kg})$ & 50 & 75 & 100 & 50 & 75 & 100 \\
\hline
\end{tabular}

Nas Figuras 3a e $3 b$ são apresentadas simulações que permitem avaliar os benefícios que se obteriam em caso de implantação da lança rotativa numa determinada planta industrial com diferentes taxas de injeção de dessulfurante.
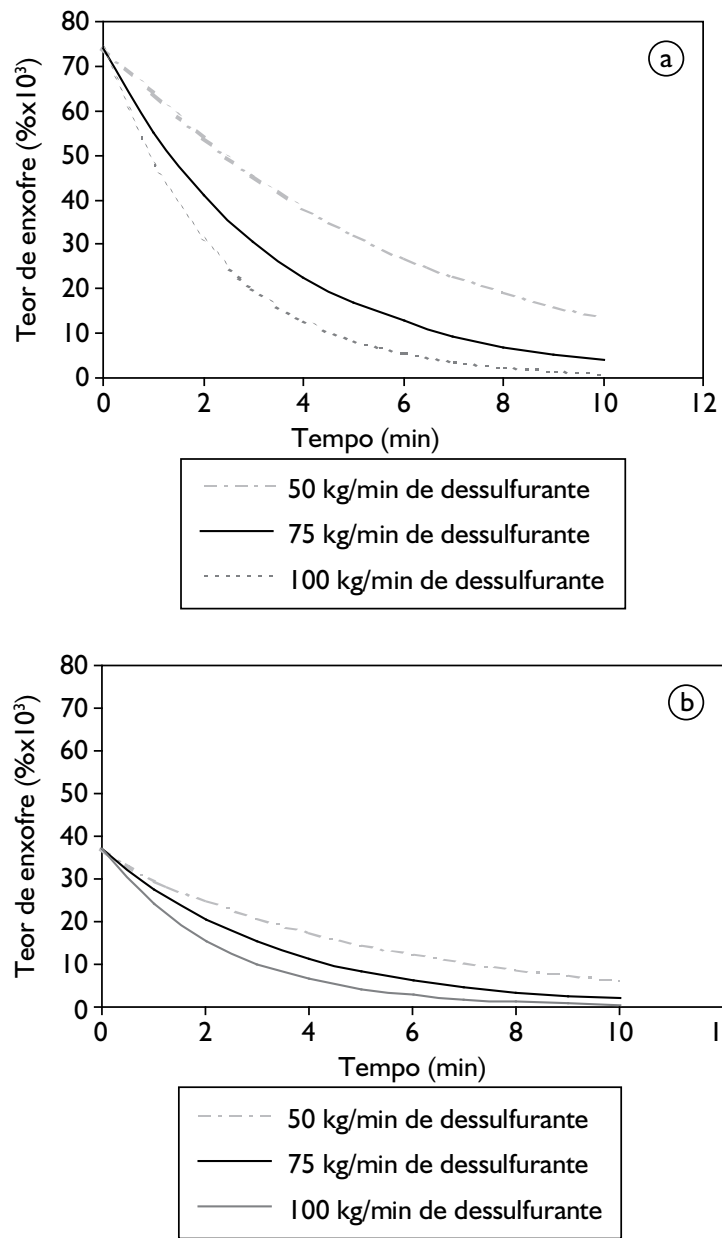

Figura 3. Evolução prevista pelo modelo de transposição.
Um primeiro benefício seria para auxiliar no estudo de viabilidade técnica e econômica de aquisição de um dispositivo para implantação da lança rotativa. Nesse caso, ter-se-ia que simular a transposição utilizando dados da própria planta de dessulfuração de gusa e comparar com os resultados que se obtém com a lança convencional, podendo-se avaliar os benefícios de produtividade e de custos.

outro benefício seria o de buscar uma melhor condição de trabalho. Conforme apresentado nas referidas figuras, um aumento da taxa de injeção de dessulfurante de $50 \mathrm{~kg} / \mathrm{min}$ para $75 \mathrm{~kg} / \mathrm{min}$ apresenta um considerável incremento na velocidade de dessulfuração do gusa. No entanto, um novo aumento para $100 \mathrm{~kg} / \mathrm{min}$ não se traduz em aumento semelhante. É importante se iniciar o tratamento de dessulfuração do gusa com teores de enxofre mais baixos para se obter os teores finais desejados em tempos menores e com economia de dessulfurante. Portanto, o modelo pode também ser utilizado para propor alterações de parâmetros operacionais com vistas à otimização de processo de dessulfuração de gusa.

\section{CONCLUSÃO}

A utilização da lança rotativa na Unidade de Dessulfuração de Gusa da Aciaria I, proporcionou um aumento de $18 \%$ na taxa de dessulfuração, uma redução de $54,5 \%$ na queda de temperatura, uma redução de $25 \%$ no consumo de agentes dessulfurantes e uma redução no tempo de tratamento de $25 \%$. Para uma mesma taxa de injeção e mesmo teor de enxofre visado, a redução do tempo de tratamento seria de I,6 min. Tudo isso permitiria uma redução do consumo de agentes dessulfurantes de $396 \mathrm{~kg}$ de cal para $317 \mathrm{~kg}$, de $88 \mathrm{~kg}$ de magnésio para $70 \mathrm{~kg}$, e de oito minutos de tratamento para 6,4 min. Além disso, a lança rotativa promove uma operação mais segura, em termos de projeção de metal líquido.

A partir dos resultados experimentais com lança rotativa na Aciaria I, determinou-se o coeficiente de transferência de massa que foi transposto para uma planta hipotética de dessulfuração de gusa, para se fazer uma série de simulações, válidas para lança rotativa.

O modelo de simulação utilizado nesse estudo pode ser aplicado para qualquer planta industrial de dessulfuração de gusa tanto para prever resultados com lança rotativa, sem avaliações experimentais prévias, quanto em estudos de otimização de plantas existentes. 


\section{REFERÊNCIAS}

I COSTA, S. L. S. Análise crítica de critérios para transposição de resultados de laboratório para a escala industrial em tratamentos de metal líquido. 1996. $132 \mathrm{f} .$. Tese (Doutorado em Metalurgia Extrativa) - Escola de Engenharia da Universidade Federal de Minas Gerais, Belo Horizonte, 1996.

2 ASAI, S.; MUCHI, I. Mass transfer rate in ladle refining process. In: INTERNATIONAL CONFERENCE ON REFINING OF IRON AND STEEL BY POWDER INJECTION, 3., 1983, Luleå, Mefos. [S.I.: s.ed.], 1983. part I, p. 12.I-12.29.

3 NAKANISHI, K. Possible relationship between energy dissipation in steel processing operation. Ironmaking \& Steelmaking, v. 2, n. 3, p. 173-97, 1975.

4 OHGUCHI, Y.; SAMBONGI, T. Kinetic study of the RH degassing process. In: MACMASTER SYMPOSIUM ON LADLE TREATMENT OF CARBON STEELS, 1979, [s.I.]. Canadá, MacMaster Academic Press; 1979. p. 9. I-9. 17.

5 WATANABE, Y. Comparison of mixing characteristics in botton blowing ladle LD converter and degassing process. Tetsu-to-Hagané, v. 69, n. 9, p.l160-765, 1983.

6 SANO, M.; MORI, K. Fluid flow and mixing characteristics in a gas-stirred molten metal bath. Transactions ISIJ, v. 23, n. 2, p. 169-75, Feb. 1983.

7 KITAMURA, S.; KITAMURA, T.; SHIBATA, T.; MIZUKAMI, Y. Effect of stirring energy temperature and flow composition on hot metal dephosphorization. ISIJ International, v. 3I, n. II, p. |322-8, I99I.

8 SZEKELY, J. Fluid flow phenomena in metal processing. New York: Academic Press, 1979.

9 SZEKELY, J; THEMELIS, N. Rate phenomena in process metallurgy. New York: John Wiley \& Sons, 1971.

10 COSTA, S.L.S.; PRENAZZI, A. ;VIANA, J.F. Dessulfuração de gusa na Usiminas. In: SEMINARIO DE ACERIA, I997, Buenos Aires. Buenos Aires: Instituto Argentino de Siderurgia, 1997. p.533-9.

Recebido em: 23/0I/07

Aceito em: 13/08/07

Proveniente de: SEMINÁRIO DE ACIARIA - INTERNACIONAL, 37., 2006, Porto Alegre. São Paulo: ABM, 2006. 